\title{
THE STIELTJES MOMENTS PROBLEM FOR RAPIDLY DECREASING FUNCTIONS
}

\author{
ANTONIO J. DURAN
}

(Communicated by J. Marshall Ash)

\begin{abstract}
We prove the following result: If $\left(a_{n}\right)_{n}$ is a sequence of complex numbers, then there exists a $\mathscr{C}^{\infty}$-function $f$ such that $f$ and all its derivatives are rapidly decreasing functions, $f(t)=0$ for $t<0$ and $\int_{0}^{+\infty} t^{n} f(t) d t=a_{n}$. We extend this result for a generalized Stieltjes moments problem. Also, we characterize the $C^{\infty}$-functions $f$ in $(0,+\infty)$ such that $f$ and all its derivatives are rapidly decreasing functions in $(0,+\infty)$ and with null moments.
\end{abstract}

\section{INTRODUCTION}

The problem of determining necessary and sufficient conditioins for a sequence of numbers $\left(a_{n}\right)_{n}$ to have the form $a_{n}=\int_{0}^{\infty} t^{n} d \alpha(t)$ where $\alpha(t)$ is non-decreasing and $n \geq 0$ was set and solved by T. J. Stieltjes in 1894-95 [12].

In 1938 R. P. Boas and G. Polya [2,9] found for each sequence $\left(a_{n}\right)_{n}$ a function $f$ of bounded variation in $(0,+\infty)$ such that $a_{n}=\int_{0}^{\infty} t^{n} d f(t)$.

We prove here that we can find a regular function $f$ with the given moments. Indeed, we prove the following result:

Theorem. Let $\left(a_{n}\right)_{n}$ be a sequence of complex numbers; then there exists a function $f$ such that:

(a) $f \in S$, where $S$ is the Schwartz's space (i.e. $f$ is a $C^{\infty}$-function and $f$ and all its derivatives are rapidly decreasing functions) and $f(t)=0$ if $t<0$.

(b) $a_{n}=\int_{0}^{\infty} t^{n} f(t) d t$ for all $n \geq 0$.

(If a function $f$ satisfies condition (a), we say that $\left.f \in S^{+} \cap S\right)$.

The key in the proof is the characterization of a certain space of analytic functions in the disc $\Omega_{0}=\left\{u \in \mathbf{C}:\left|u-\frac{1}{2}\right|<\frac{1}{2}\right\}$ (this space is obtained from

Received by the editors March 3, 1989. Part of this paper has been presented in the Congreso de Analisis Funcional (Madrid, June 1988) and in the XIV Jornadas Hispano Lusas de Matematicas (Puerto de la Cruz, June 1989). Part of this work forms part of the author's Ph.D. Thesis prepared at the Universidad de Sevilla under the direction of Professor J. Arias.

1980 Mathematics Subject Classification (1985 Revision). Primary 44A60.

Key words and phrases. Moments problem, rapidly decreasing functions. 
$S^{+} \cap S$ by taking Fourier transform in a suitable sense) in which an interpolation theorem is proved (see $\S 2$ ).

This characterization is obtained from the results in [3], where we study the Fourier coefficients of functions in the space $S^{+} \cap S$ with respect to a certain orthonormal system. We relate the coefficients of a function with its Fourier transform (see $\S 1)$.

In $\S 4$, using certain properties obtained in [3] for a Hankel transform (which was used by Hardy [8] to study a Stieltjes moments problem) defined by

$$
\mathscr{H}_{0}(f)(t)=\frac{1}{2} \int_{0}^{\infty} f(x) J_{0}(\sqrt{x t}) d x,
$$

where $J_{0}$ is the Bessel function of first kind and $f$ is a suitable function, we characterize in a unexpected way (because of its simplicity), the functions $f$ defined on $(0,+\infty)$, such that $f$ is a $C^{\infty}$-function and $f$ and all its derivatives are rapidly decreasing in $(0,+\infty)$ (we shall say that $f \in S^{+}$) with null moments. Indeed, we prove:

Theorem. Let $\phi$ be a $C^{\infty}$-function in $(0,+\infty)$ such that $\sup _{t>0} t^{k}\left|\phi^{(n)}(t)\right|<\infty$ for all $k, n \geq 0$. Then $\int_{0}^{\infty} t^{n} \phi(t) d t=0$ if and only if there exists a function $\psi \in S^{+} \cap S$ such that $\phi(t)=\frac{1}{2} \int_{0}^{\infty} \psi(x) J_{0}(\sqrt{x t}) d x$.

Also, in $\S 4$ we propose a problem on the functions of $S^{+} \cap S$ with null moments. Finally, we extend the previous results to a generalized moment problem.

Terminology. Throughout the paper $S$ denotes the Schwartz's space, i.e. $f \in S$ if and only if $f$ is a $C^{\infty}$-function and $f$ and all its derivatives are rapidly decreasing functions. We write $s$ for the space of rapidly decreasing sequences. The Fourier transform of a function $f$ is denoted by $\hat{f}$. We use the following standard notation for special functions: the Laguerre polynomials are denoted by $L_{n}^{\alpha}(t)=\sum_{k=0}^{n}\left(\begin{array}{c}n+\alpha \\ n-k\end{array}\right)(-t)^{k} / k !, \alpha>-1$, and the Bessel function of first kind by $J_{0}$. We write $D$ for the unit disc $D=\{w \in \mathbf{C}:|w|<1\}$ and $\Omega_{0}$ for the $\operatorname{disc} \Omega_{0}=\left\{u \in \mathbf{C}:\left|u-\frac{1}{2}\right|<\frac{1}{2}\right\}$.

\section{Preliminaries}

We shall consider the space

$$
\begin{aligned}
S^{+}= & \left\{\phi:(0,+\infty) \rightarrow \mathbf{C} \text { such that } \phi \text { is a } C^{\infty}\right. \text {-function and } \\
& \left.\|\phi\|_{k, n}=\sup _{t \in(0,+\infty)} t^{k}\left|\phi^{(n)}(t)\right|<+\infty \text { for all } k, n \in \mathbf{N}\right\}
\end{aligned}
$$

It is easy to verify that $\left(S^{+},\|\cdot\|_{k, n}, k, n \in \mathbf{N}\right)$ is a Fréchet space, and that $\phi \in S^{+}$if and only if $\phi$ is the restriction to $(0,+\infty)$ of a function of the Schwartz's space $S$.

Also, we shall consider the following space: $S^{+} \cap S=\{\phi \in S: \phi(t)=0$ for $t<0\}$, which can be considered as a subspace of $S^{+}$, in a natural way. It is 
easy to prove that if $\phi \in S^{+}$then $\phi \in S^{+} \cap S$ if and only if $\lim _{t \rightarrow 0^{+}} \phi^{(k)}(t)=0$ for all $k \geq 0$.

In [3, Th. 2.9] or [7, p. 548], are defined the Fourier-Laguerre coefficients of a function $\phi \in S^{+}$by $a_{n}=\int_{0}^{+\infty} \phi(t) L_{n}(t) e^{-t / 2} d t$ with $n \geq 0$ and there is proved the following theorem:

Theorem A. The mapping $\mathscr{L}: S^{+} \rightarrow s$ defined by

$$
\mathscr{L}(\phi)=\left(a_{n}\right)_{n}=\left(\int_{0}^{+\infty} \phi(t) L_{n}(t) e^{-t / 2} d t\right)_{n}
$$

is an isomorphism from $\mathrm{S}^{+}$onto $s$.

In [3, Th. 3.2], we characterize the Fourier-Laguerre coefficients of the functions of the space $S^{+} \cap S$ in the following way:

Theorem B. Let $\phi \in S^{+}$and $a_{n}=\int_{0}^{+\infty} \phi(t) L_{n}(t) e^{-t / 2} d t$. Then $\phi \in S^{+} \cap S$ if and only if $\sum_{n=0}^{\infty} n^{k} a_{n}=0$ for all $k \geq 0$.

Also in [3, Th. 3.5] we prove the following theorem that we shall use later:

Theorem C. The integral operator $\mathscr{H}_{0}: S^{+} \rightarrow S^{+}$, defined by

$$
\mathscr{H}_{0}(\phi)(x)=\frac{1}{2} \int_{0}^{\infty} \phi(t) J_{0}(\sqrt{x t}) d t
$$

is an isomorphism of $S^{+}$onto itself, and $\mathscr{H}_{0}^{2}$ is the identity map. Moreover, if $\left(a_{n}\right)_{n}$ are the Fourier-Laguerre coefficients of $\phi$ then $\left((-1)^{n} a_{n}\right)_{n}$ are the FourierLaguerre coefficients of $\mathscr{H}_{0}(\phi)$.

The operator $\mathscr{H}_{0}$ is called a Hankel transform of degree 0.

\section{Some ANALYTiC FUnCtions SPACES RELATED to $S^{+}$}

Let $\phi \in L^{1}([0,+\infty))$, and consider the following analytic function $\widetilde{\phi}$ in the lower half plane:

$$
\widetilde{\phi}(z)=\int_{0}^{+\infty} \phi(t) e^{-2 \pi i z t} d t .
$$

(Notice that if $z \in \mathbf{R}$ then $\widetilde{\phi}(z)=\widehat{\phi}(z)$.) Using the bilinear transformation $W(z)=\left(-\frac{1}{2}+2 \pi i z\right) /\left(\frac{1}{2}+2 \pi i z\right)$, which transforms the lower half plane in the unit disc, we obtain the following analytic function $\check{\phi}$ in the unit disc:

$$
\check{\phi}(w)=\tilde{\phi}(Z(w))=\int_{0}^{\infty} \phi(t) e^{-\frac{1}{2}\left(\frac{1+w}{1-w}\right) t} d t .
$$

In the following theorem we relate the function $\check{\phi}$ with the Fourier-Laguerre coefficients of $\phi$, for $\phi \in S^{+}$. 
Theorem 2.1. Let $\phi \in S^{+}$and $a_{n}=\int_{0}^{\infty} \phi(t) L_{n}(t) e^{-t / 2} d t$. Then $\check{\phi}(w)=$ $(1-w) \sum_{n=0}^{\infty} a_{n} w^{n}$ for $|w|<1$.

Proof. As $\left(L_{n}(t) e^{-t / 2}\right)_{n}$ is an orthonormal system in $L^{2}([0,+\infty)), \phi(t)=$ $\sum_{n} a_{n} L_{n}(t) e^{-t / 2}$ and the series converges in $L^{2}([0,+\infty))$. Since $e^{-2 \pi i z t} \in$ $L^{2}([0,+\infty))$ if $\mathfrak{I} z<0$, we obtain $\widetilde{\phi}(z)=\sum_{n} a_{n} \int_{0}^{\infty} L_{n}(t) e^{-t / 2} e^{-2 \pi i z t} d t$. From [6, p. 191] we get $\widetilde{\phi}(z)=\sum_{n} a_{n}\left(-\frac{1}{2}+2 \pi i z\right)^{n} /\left(\frac{1}{2}+2 \pi i z\right)^{n+1}$. If we put $w=\left(-\frac{1}{2}+2 \pi i z\right) /\left(\frac{1}{2}+2 \pi i z\right)$ we obtain $\check{\phi}(w)=(1-w) \sum_{n} a_{n} w^{n}$.

Considering Theorems A, B and 2.1 we obtain the following characterizations:

Theorem 2.2. Let $f \in H(D)$. The following conditions are equivalent:

(a) There exists $\phi \in S^{+}$such that $f(w)=\check{\phi}(w)$.

(b) $f(w) /(1-w)$ and all its derivatives are bounded on $D$.

Theorem 2.3. Let $f \in H(D)$. The following conditions are equivalent:

(a) There exists $\phi \in S^{+} \cap S$ such that $f(w)=\check{\phi}(w)$.

(b) $f(w) /(1-w)$ and all its derivatives are bounded on $D$ and $\lim _{w \rightarrow 1}(f(w) /(1-w))^{(k)}=0$ for all $k \geq 0$.

Let $\phi \in S^{+}$. Given $\phi(t) e^{t / 2}$, in a similar way as we did for $\phi$, we can define two functions $\left(\phi(t) e^{t / 2}\right)^{\sim}(z)$ and $\left(\phi(t) e^{t / 2}\right)^{-(}(u)$, which are analytic in $\{z \in \mathbf{C}: \mathfrak{I} z<-1 / 4 \pi\}$ and $\Omega_{0}=\left\{u \in \mathbf{C}:\left|u-\frac{1}{2}\right|<\frac{1}{2}\right\}$ respectively.

Theorem 2.4. Let $f \in H\left(\Omega_{0}\right)$. The following conditions are equivalent:

(a) There exists $\phi \in S^{+} \cap S$ such that $\left(\phi(t) e^{t / 2}\right)(u)=f(u)$.

(b) $f(u) /(1-u)$ and all its derivatives are bounded on $\Omega_{0}$ and $\lim _{u \rightarrow 1}(f(u) /(1-u))^{(k)}=0$ for all $k \geq 0$.

Proof. (a) $\Rightarrow(\mathrm{b})$. We define $\psi(t)=\phi(t) e^{t / 2}$ and $g(z)=\tilde{\psi}(z-i / 4 \pi)$. It is clear that $g(z)=\widetilde{\phi}(z)$. Let $h \in H\left(\Omega_{0}\right)$ defined by $h(u)=\check{\phi}(3-(4 /(u+1)))$ (it is well defined because $w(u)=3-(4 /(u+1))$ transforms $\Omega_{0}$ in $\left.D\right)$. By Theorem 2.3 (and because $d^{k} w(u) / d u^{k}$ is bounded in $\Omega_{0}$ for all $\left.k \geq 0\right) h(u) /(1-u)$ and all its derivatives are bounded in $\Omega_{0}$ and $\lim _{u \rightarrow 1}(h(u) /(1-u))^{(k)}=0$ for all $k \geq 0$. But

$$
h(u)=\check{\phi}(w(u))=\widetilde{\phi}(Z(w(u)))=g(Z(w(u)))=\widetilde{\psi}\left(\frac{1}{4 \pi i} \frac{1+u}{1-u}\right)=\check{\psi}(u)=f(u)
$$

and the proof is finished.

(b) $\Rightarrow($ a). Follows similarly because $u(w)=4 /(3-w)-1$ transforms $D$ in $\Omega_{0}$ and $d^{k} u(w) / d w^{k}$ is bounded in $D$ for all $k \geq 0$.

We need the next theorem, whose proof is suggested by Gardins proof of a classical Borel's Theorem (see [4, p. 50]). 
Theorem 2.5. Let $\left(a_{n}\right)_{n}$ be a sequence of complex numbers. There exists a function $f \in H\left(\Omega_{0}\right)$ such that:

(a) $f$ and all its derivatives are bounded in $\Omega_{0}$.

(b) $\lim _{u \rightarrow 0} f^{(n)}(u)=a_{n}$ for all $n \geq 0$.

(c) $\lim _{u \rightarrow 1} f^{(n)}(u)=0$ for all $n \geq 0$.

We need two lemmas:

Lemma 1. There exists an analytic function $F: \Omega_{0} \rightarrow \mathrm{C}$ satisfying:

(a) $F$ and all its derivatives are bounded in $\Omega_{0}$.

(b) $\lim _{u \rightarrow 0} F^{(n)}(u)=0$ for all $n>0$ and $\lim _{u \rightarrow 0} F(u)=1$.

(c) $\lim _{u \rightarrow 1} F^{(n)}(u)=0$ for all $n \geq 0$

Proof. Let $\phi \in S^{+} \cap S$ and $a_{n}=\int_{0}^{\infty} \phi(t) L_{n}(t) e^{-t / 2} d t$. By Theorem 2.3, $\dot{\phi}(w)$ and all its derivatives are bounded in $D$ and $\lim _{w \rightarrow 1} \check{\phi}^{(n)}(w)=0$ for all $n \geq 0$. We can choose $\phi$ such that $a_{0} \neq 0$ and $\sup _{w \in D}|\dot{\phi}(w)|=1$, and there exists $z_{0} \neq 1,\left|z_{0}\right|=1$ such that $\lim _{w \rightarrow z_{0}} \check{\phi}(w)=1$. Let $b_{0}=0$ and $b_{n}=a_{n}$ if $n \geq 1$. We consider the function $h(w)=\sum_{n} b_{n} w^{n}$. Since $\left(b_{n}\right) \in s, h$ and all its derivatives are bounded in $D$. Also $\lim _{w \rightarrow 0} h(w)=0$ and by the choice of the coefficients (notice that by Theorem B $\sum_{n} n^{k} a_{n}=0$ for all $k \geq 0$ ) $\lim _{w \rightarrow 1} h(w)=-a_{0} \neq 0$ and $\lim _{w \rightarrow 1} h^{(n)}(w)=0$ for all $n \geq 1$.

Let $g: D \rightarrow \mathbf{C}$ be defined by $g(w)=h(\check{\phi}(w)$ ) (it is well defined because $|\check{\phi}(w)| \leq 1$ for all $w \in D)$. Notice that $g$ and all its derivatives are bounded in $D$.

We consider a bilinear application $H$, which transforms $\Omega_{0}$ in $D$, sending 0 into $z_{0}$ and 1 into 1 .

Finally, we take $F: \Omega_{0} \rightarrow \mathbf{C}$ defined by $F(u)=-\left(1 / a_{0}\right) g(H(u))$.

Lemma 2. Let $u \in \Omega_{0}$. Given $n$, let $z$ be such that $z^{2^{n}}=u$ and $|\arg (z)|$ is the smallest possible. Then $z \in \Omega_{0}$.

Proof. It is enough to prove the Lemma for $\left|u-\frac{1}{2}\right|=\frac{1}{2}$. We then write $u=$ $1 /\left(1+\tan ^{2}(\theta)\right)+\left(\tan \theta /\left(1+\tan ^{2} \theta\right)\right) i$ with $-\pi / 2<\theta<\pi / 2$, so that $\left|z-\frac{1}{2}\right| \leq \frac{1}{2}$ is equivalent to $(\cos (\theta))^{1 / 2^{n}} \leq \cos \left(\theta / 2^{n}\right)$, and we proceed by induction.

Proof ( of Theorem 2.5). Let $\left(a_{n}\right)_{n}$ be a sequence of complex numbers. We define the sequence $\left(b_{n}\right)_{n}$ as $b_{n}=1 / 2^{k_{n}}$ with $k_{n}=\min \left\{m: 1 / 2^{m}<1 /\left(\left[\left|a_{n}\right|\right]+\right.\right.$ 1)\} (where $[x]$ is the integral part of the real number $x$ ). We define the function $f: \Omega_{0} \rightarrow \mathbf{C}$ as

$$
f(u)=\sum_{n=0}^{\infty} \frac{a_{n}}{n !} u^{n} F\left(u^{b_{n}}\right),
$$

where $F$ is the same function in Lemma 1 and we choose $u^{b_{n}}$ as in Lemma 2 (with this choice $u^{b_{n}}$ is an analytic function in $\Omega_{0}$, and take its values in $\Omega_{0}$ ).

We prove that $f$ and all its derivatives are bounded in $\Omega_{0}$. 
Since $\lim _{u \rightarrow 1} F^{(n)}(u)=0$ for all $n \geq 0$, there exists $M_{1}>0$ such that $|F(u)| \leq M_{1}|1-u|^{2}$. Let $u$ be such that $\left|u-\frac{1}{2}\right|=\frac{1}{2}$; we can write $u=$ $1 /\left(1+\tan ^{2}(\theta)\right)+\left(\tan \theta /\left(1+\tan ^{2}(\theta)\right) i\right.$ with $-\pi / 2<\theta<\pi / 2$. It is clear that $|1-u|^{2}=\sin ^{2}(\theta)$ and $1-|u|=1-\cos (\theta)$. So $|F(u)| \leq M(1-|u|)$ for $u \in \Omega_{0}$.

The following shows that $f$ is analytic and bounded in $\Omega_{0}$ :

$$
\left|\frac{a_{n}}{n !} u^{n} F\left(u^{b_{n}}\right)\right| \leq \frac{\left|a_{n}\right|}{n !}|u|^{n} M\left(1-|u|^{b_{n}}\right) \leq M \frac{\left|a_{n}\right|}{n !} b_{n}|u|^{n+b_{n}-1} \leq M \frac{1}{n !}
$$

for all $u \in \Omega_{0}$ and $n \geq 1$;

and from $n=0\left|F\left(u_{b_{0}}\right)\right| \leq$ constant.

We shall study the derivatives of $f$.

We have:

$$
\begin{aligned}
f^{(k)}(u)= & \sum_{n=k}^{\infty} \frac{a_{n}}{n !} n(n-1) \cdots(n-k+1) u^{n-k} F\left(u^{b_{n}}\right) \\
& +\sum_{m=1}^{k}\left(\begin{array}{c}
k \\
m
\end{array}\right) \sum_{n=k-m}^{\infty} \frac{a_{n}}{n !} n(n-1) \cdots(n-k+m+1) u^{n-k+m} \frac{d^{m} F\left(u^{b_{n}}\right)}{d u^{m}} \\
= & \sum_{n=0}^{\infty} \frac{a_{n+k}}{n !} u^{n} F\left(u^{b_{n+k}}\right)+\sum_{m=1}^{k}\left(\begin{array}{c}
k \\
m
\end{array}\right) \sum_{n=0}^{\infty} \frac{a_{n+k-m}}{n !} u^{n} \frac{d^{m} F\left(u^{b_{n+k-m}}\right)}{d u^{m}} .
\end{aligned}
$$

Proceeding as in (1), we obtain

$$
\left|\frac{a_{n+k}}{n !} u^{n} F\left(w^{b_{n+k}}\right)\right| \leq M_{2} \frac{1}{n !} \quad \text { if } u \in \Omega_{0} \text { for certain } M_{2}>0
$$

We can write (see $[11$, p. $12(83)])$

$$
\begin{aligned}
u^{n} \cdot \frac{d^{m} F\left(u^{b_{n+k-m}}\right)}{d u^{m}}= & u^{n} \cdot \sum_{l=1}^{m} \frac{(-1)^{l}}{l !} \sum_{j=1}^{l}(-1)^{j}\left(\begin{array}{l}
l \\
j
\end{array}\right) u^{(l-j) b_{n+k-m}} j b_{n+k-m} \\
& \left(j b_{n+k-m}-1\right) \cdots\left(j b_{n+k-m}-m+1\right) u^{j b_{n+k-m-m}} F^{(l)}\left(u^{b_{n+k-m}}\right) \\
= & b_{n+k-m} \sum_{l=1}^{m} \frac{(-1)^{l}}{l !} \sum_{j=1}^{l}(-1)^{j}\left(\begin{array}{l}
l \\
j
\end{array}\right) j\left(j b_{n+k-m}-1\right) \cdots \\
& \cdots\left(j b_{n+k-m}-m+1\right) u^{l b_{n+k-m}} \frac{F^{(l)}\left(u^{b_{n+k-m}}\right)}{u^{m-n}} .
\end{aligned}
$$

If $n \geq m$, since $F$ and all its derivatives are bounded in $\Omega_{0}$, we obtain $\left|F^{(l)}\left(u^{b_{n+k-m}}\right) / u^{m-n}\right| \leq C_{1}$ if $u \in \Omega_{0}$ (the constant only depends on $l$ ).

If $0 \leq n<m$, let $\beta=\max \left\{\left[(m-n) / b_{n+k-m}\right]+1: 0 \leq n<m\right\}$. Since $\lim _{u \rightarrow 0} F^{(\alpha)}(u)=0$ if $\alpha \geq 1$, there exists $C_{2}>0$, (which only depends on $l, m$ and $k$ ) such that $\left|F^{(l)}(u)\right| \leq C_{2}|u|^{\beta}$ if $u \in \Omega_{0}$. So

$$
\left|\frac{F^{(l)}\left(u^{b_{n+k-m}}\right)}{u^{m-n}}\right| \leq C_{2} \frac{|u|^{\beta b_{n+k-m}}}{|u|^{m-n}} \leq C_{2}
$$


if $u \in \Omega_{0}$. So, for $n \geq 0,\left|F^{(l)}\left(u^{b_{n+k-m}}\right) / u^{m-n}\right| \leq$ constant if $u \in \Omega_{0}$, and the constant only depends on $l, m$ and $k$. Hence $\left|u^{n} \cdot d^{m} F\left(u^{b_{n+k-m}}\right) / d u^{m}\right| \leq$ $M_{3} b_{n+k-m}$ if $u \in \Omega_{0}$, where $M_{3}$ is a positive constant, $m=1, \ldots, k$ and $n \geq 0$.

We obtain

$$
\left|\frac{a_{n+k-m}}{n !} u^{n} \frac{d^{m} F\left(u^{b_{n+k-m}}\right)}{d u^{m}}\right| \leq \frac{M_{3}}{n !} \quad \text { if } u \in \Omega_{0} .
$$

Using (2), (3) and (4), we get $\left|f^{(k)}(u)\right| \leq$ constant if $u \in \Omega_{0}$.

By the properties of $F$ and $(2), \lim _{u \rightarrow 0} f^{(k)}(u)=a_{k}$ and $\lim _{u \rightarrow 1} f^{(k)}(u)=0$ for all $k \geq 0$, and the proof is finished.

In [14] using other techniques, we can find theorems of the same kind as Theorem 2.5 for more general sets $\Omega$, but the function $f$ which interpolates the sequence $\left(a_{n}\right)_{n}$ does not satisfy condition (c).

\section{The Stieltjes moments problem in the SPace $S^{+} \cap S$}

We prove that the Stieltjes moments problem always has a solution in the space $S^{+} \cap S$ (and so in the space $S^{+}$).

Theorem 3.1. Let $\left(a_{n}\right)_{n}$ be a sequence of complex numbers. Then there exists a function $\phi \in S^{+} \cap S$ such that $\int_{0}^{\infty} t^{n} \phi(t) d t=a_{n}$ for all $n \geq 0$.

Proof. We define the following sequence $b_{n}=\sum_{k=0}^{n}\left((-1)^{k} / k !\right)\left(\begin{array}{l}n \\ k\end{array}\right) a_{k}$. As $L_{n}(t)=\sum_{k=0}^{n}\left((-1)^{k} / k !\right)\left(\begin{array}{l}n \\ k\end{array}\right) t^{k}$, if there exists $\phi \in S^{+} \cap S$ such that $\int_{0}^{\infty} \phi(t) L_{n}(t) d t=b_{n}$, it is clear that $\int_{0}^{\infty} \phi(t) t^{n} d t=a_{n}$. Let $c_{n}=n ! b_{n}$. By Theorems 2.4 and 2.5, there exists $f \in H\left(\Omega_{0}\right)$ and $\phi \in S^{+} \cap S$ such that $g(u)=$ $(1-u) f(u)=\left(\phi e^{t / 2}\right)^{-}(u)$ and $\lim _{u \rightarrow 0} f^{(n)}(u)=c_{n}$. We shall prove that $b_{n}=$ $\int_{0}^{+\infty} \phi(t) L_{n}(t) d t$. Indeed

$$
\begin{aligned}
g(u) & =\int_{0}^{+\infty} \phi(t) e^{-t\left(\frac{u}{1-u}\right)} d t \\
& =(1-u) \int_{0}^{+\infty} \phi(t) \frac{1}{1-u} e^{-t\left(\frac{u}{1-u}\right)} d t \\
& =(1-u) \int_{0}^{+\infty} \varphi(t, u) d t .
\end{aligned}
$$

So $f(u)=\int_{0}^{+\infty} \varphi(t, u) d t$.

By [6, p. 189] $\varphi(t, u)=\phi(t) \sum_{k=0}^{\infty} L_{k}(t) u^{k}$, for $t \in \mathbf{R}$ and $|u|<1$.

Formally

$$
f^{(n)}(u)=\int_{0}^{+\infty} \phi(t) \sum_{n=k}^{\infty} k(k-1) \cdots(k-n+1) L_{k}(t) u^{k-n} d t
$$

and

$$
\lim _{u \rightarrow 0} f^{(n)}(u)=\int_{0}^{+\infty} \phi(t) n ! L_{n}(t) d t,
$$

and as $\lim _{u \rightarrow 0} f^{(n)}(u)=c_{n}$ we deduce that $b_{n}=\int_{0}^{+\infty} \phi(t) L_{n}(t) d t$. 
Now it suffices to see that $|\varphi(t, u)| \leq h(t)$ with $h \in L^{1}([0,+\infty))$. As

$$
\begin{aligned}
\varphi(t, u) & =\phi(t) \frac{1}{1-u} \exp \left(-t \frac{u}{1-u}\right) \\
& =\phi(t) \frac{1}{1-u} \exp \left(-t \frac{(x+i y)(1-x+i y)}{(1-x)^{2}+y^{2}}\right)
\end{aligned}
$$

with $u=x+i y$, we have $|\varphi(t, u)| \leq\left|\phi(t) \frac{1}{1-u}\right| \exp \left(-t \frac{x(1-x)-y^{2}}{(1-x)^{2}+y^{2}}\right)$.

So is enough to take $u \in \Omega_{0}$ with $\Re u<\frac{1}{2}$.

We deduce the existence of solution for a generalized Stieltjes moments problem.

Corollary 3.2. Let $\left(a_{n}\right)_{n}$ be an sequence of complex numbers and let $\left(k_{n}\right)_{n}$ be an arithmetic sequence of real numbers (i.e. $k_{n}=a+b n$ for $a, b \in \mathbf{R}$ ). Then there exists a function $\phi \in S^{+} \cap S$ such that $\int_{0}^{\infty} t^{k_{n}} \phi(t) d t=a_{n}$ for all $n \geq 0$.

Proof. By the previous theorem, there exists $\phi \in S^{+} \cap S$ such that $\int_{0}^{\infty} t^{n} \phi(t) d t=$ $a_{n}$ for all $n \geq 0$. Setting $t=x^{b}$, we get $a_{n}=\int_{0}^{\infty}|b| x^{-a+b-1} \phi\left(x^{b}\right) x^{k_{n}} d x$. Notice that as $\phi \in S^{+} \cap S$ then $\lim _{t \rightarrow 0} \phi^{(k)}(t)=0$ for all $k \geq 0$, so $\psi(x)=$ $|b| x^{-a+b-1} \phi\left(x^{b}\right) \in S^{+} \cap S$ and $\psi(x) x^{k_{n}} \in L^{1}([0,+\infty))$, which proves the theorem.

\section{FUnCtions IN $S^{+}$WITH NULL MOMENTS}

In the following theorem, we characterize the functions of $S^{+}$such that their moments are null.

Theorem 4.1. Let $\phi \in S^{+}$, and $a_{n}=\int_{0}^{\infty} \phi(t) L_{n}(t) e^{-t / 2} d t$. The following conditions are equivalent:

(a) $\int_{0}^{\infty} t^{k} \phi(t) d t=0$ for all $k \geq 0$.

(b) $\sum_{n}(-1)^{n} n^{k} a_{n}=0$ for all $k \geq 0$.

(c) There exists $\psi \in S^{+} \cap S$ such that $\phi$ is the Hankel transform of degree 0 of $\psi$, i.e.

$$
\phi(t)=\mathscr{H}_{0}(\psi)(t)=\frac{1}{2} \int_{0}^{\infty} \psi(x) J_{0}(\sqrt{x t}) d x
$$

Proof. (a) $\Rightarrow$ (b). It is clear that $\int_{0}^{\infty} \phi(t) t^{k} d t=0$ for all $k \geq 0$ if and only if $\lim _{z \rightarrow 0} \widetilde{\phi}^{(k)}(z)=0$ for all $k \geq 0$ and so if and only if $\lim _{w \rightarrow-1} \check{\phi}^{(k)}(w)=0$ for all $k \geq 0$ (because $\check{\phi}(w)=\tilde{\phi}(Z(w))$ and $d^{n} Z(w) / d w^{n}$ is bounded near $w=-1)$. It is clear that $\lim _{w \rightarrow-1} \check{\phi}^{(k)}(w)=0$ for all $k \geq 0$ if and only if $\lim _{w \rightarrow-1}(\check{\phi}(w) /(1-w))^{(k)}=0$. We have $\dot{\phi}(w)=(1-w) \sum_{n} a_{n} w^{n}$ and 
$\left(a_{n}\right)_{n} \in s$, so $\check{\phi}(w) /(1-w)=\sum_{n} a_{n} w^{n}$ and $\left(\frac{\check{\phi}(w)}{(1-w)}\right)^{(k)}=\sum_{n} n^{k} a_{n} w^{n-k}+b_{k-1}^{(k)} \sum_{n} n^{k-1} a_{n} w^{n-k}+\cdots+b_{1}^{(k)} \sum_{n} n a_{n} w^{n-k}$

for certain $b_{k-1}^{(k)}, \ldots, b_{1}^{(k)} \in \mathbf{N}$ and $k \geq 1$. Applying successively Theorems of Tauber and Abel, we get $\int_{0}^{\infty} \phi(t) t^{k} d t=0$ if and only if $\sum_{n}(-1)^{n} n^{k} a_{n}=0$ for all $k \geq 0$.

(b) $\Leftrightarrow\left(\right.$ c). By Theorem $C$ the Fourier-Laguerre coefficients of $\mathscr{H}_{0}(\phi)$ are $\left((-1)^{n} a_{n}\right)_{n}$. So by Theorem B $\sum_{n}(-1)^{n} n^{k} a_{n}=0$ if and only if $\mathscr{H}_{0}(\phi) \in S^{+} \cap S$. Since $\mathscr{H}_{0}^{2}=\mathrm{id}$, setting $\psi=\mathscr{H}_{0}(\phi)$ we finish the proof.

We shall extend the previous theorem for the generalized moment problem. In [3] Theorem $\mathrm{A}$ is generalized in the following way:

Let $\alpha>-1, t^{\alpha / 2} S^{+}=\left\{t^{\alpha / 2} \phi: \phi \in S^{+}\right\}$and $\mathscr{L}_{n}^{\alpha}(t)=\tau_{n} L_{n}^{\alpha}(t) t^{\alpha / 2} e^{-t / 2}$, where $\tau_{n}=(n ! / \Gamma(n+\alpha+1))^{1 / 2}$ and $L_{n}^{\alpha}(t)$ are the generalized Laguerre polynomials. It is well known that $\left(\mathscr{L}_{n}^{\alpha}(t)\right)_{n}$ is an orthonormal system in $L^{2}([0,+\infty))$. If $\psi \in t^{\alpha / 2} S^{+}$we define the Fourier-Laguerre coefficients of $\psi$ as $a_{n}=$ $\int_{0}^{\infty} \psi(t) \mathscr{L}_{n}^{\alpha}(t) d t$. We prove in [3] the following theorem:

Theorem D. The mapping $\mathscr{L}: t^{\alpha / 2} S^{+} \rightarrow s$ defined by

$$
\mathscr{L}(\phi)=\left(a_{n}\right)_{n}=\left(\int_{0}^{+\infty} \phi(t) \mathscr{L}_{n}^{\alpha}(t) d t\right)_{n}
$$

is an isomorphism of the space $t^{\alpha / 2} S^{+}$onto $s$.

The proofs of the following theorems are analogous to the proofs of Theorems 2.1 and 2.2 .

Theorem 4.2. Let $\phi \in S^{+}$and $a_{n}=\int_{0}^{\infty} t^{\alpha / 2} \phi(t) \mathscr{L}_{n}^{\alpha}(t) d t$. Then $\left(t^{\alpha} \phi\right)^{-}(w)=$ $(1-w)^{\alpha+1} \sum_{n=0}^{\infty} a_{n} \tau_{n}^{-1} w^{n}$.

Theorem 4.3. Let $f \in H(D)$. The following are equivalent:

(a) There exists $\phi \in S^{+}$such that $f(w)=\left(t^{\alpha} \phi\right)^{-}(w)$.

(b) $f(w) /(1-w)^{\alpha+1}$ and all its derivatives are bounded on $D$.

Applying these results, we obtain:

Theorem 4.4. Let $\alpha>0$ and $\phi \in S^{+}$. Then $\int_{0}^{\infty} t^{\alpha+n} \phi(t) d t=0$ if and only if there exists $\psi \in S^{+} \cap S$ such that $\phi(t)=\left(1 / t^{\alpha}\right)\left(\mathscr{H}_{0}(\psi) *\left(x^{\alpha-1} e^{-x / 2} / \Gamma(\alpha)\right)(t)\right.$.

Proof. Proceeding as in the proof of Theorem 4.1 we obtain $\int_{0}^{\infty} t^{\alpha+n} \phi(t) d t=0$ if and only if $\lim _{u \rightarrow-1}\left(t^{\alpha} \phi\right)^{(\tilde{k})}(w)=0$ for all $k \geq 0$. So $\lim _{w \rightarrow-1}\left(\left(t^{\alpha} \phi\right)^{-}(w) /\right.$ $\left.(1-w)^{\alpha+1}\right)^{(k)}(w)=0$ for all $k \geq 0$. 
By Theorem $4.2\left(t^{\alpha} \phi\right)^{-}(w) /(1-w)^{\alpha+1}=\sum_{n} b_{n} w^{n}$ with $\left(b_{n}\right) \in s$, so $\int_{0}^{\infty} t^{\alpha+n} \phi(t) d t=0$ if and only if $\sum_{n}(-1)^{n} n^{k} b_{n}=0$ for all $k \geq 0$ (see proof of Theorem 4.1).

By Theorems $\mathrm{A}$ and 4.1, we obtain $\int_{0}^{\infty} t^{\alpha+n} \phi(t) d t=0$ if and only if $\left(t^{\alpha} \phi\right)^{-}(w)=(1-w)^{\alpha}\left(\mathscr{H}_{0}(\psi)\right)(w)$ where $\psi \in S^{+} \cap S$. If we put $w=\left(-\frac{1}{2}+\right.$ $2 \pi i z) /\left(\frac{1}{2}+2 \pi i z\right)$, it follows that

$$
\widetilde{t^{\alpha}} \phi(z)=\frac{1}{\left(\frac{1}{2}+2 \pi i z\right)^{\alpha}} \widetilde{\mathscr{H}_{0}(\psi)}(z) .
$$

If $z \in \mathbf{R}$ and noticing that $\left(x^{\alpha-1} e^{-x / 2} / \Gamma(\alpha)\right)^{\wedge}(z)=1 /\left(\frac{1}{2}+2 \pi i z\right)^{\alpha}$ (see [5, p. 144]) we get

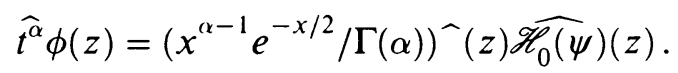

So $t^{\alpha} \phi(t)=\left(\left(x^{\alpha-1} e^{-x / 2} / \Gamma(\alpha)\right) * \mathscr{H}_{0}(\psi)\right)(t)$ and the theorem is proved.

We study the functions of $S^{+} \cap S$ with null moments.

Let $T=\left\{\phi \in S^{+} \cap S: \int_{0}^{\infty} t^{k} \phi(t) d t=0\right.$ for all $\left.k \geq 0\right\}$. From Theorems B, C and 4.1 we get:

Theorem 4.5. Let $\phi \in S^{+}$and $a_{n}=\int_{0}^{\infty} \phi(t) L_{n}(t) e^{-t / 2} d t$. Then $\phi \in T$ if and only if $\sum_{n} n^{k} a_{n}=0$ and $\sum_{n}(-1)^{n} n^{k} a_{n}=0$ for all $k \geq 0$. So $T=$ $\left(S^{+} \cap S\right) \cap \mathscr{H}_{0}\left(S^{+} \cap S\right)$ and $\mathscr{H}_{0}$ is an isomorphism of $T$ onto itself.

We give examples of non-null functions in the spaces $T$.

Indeed, let $\phi_{1}, \phi_{2} \in S^{+} \cap S$ and consider $\phi_{3}=\phi_{1} * \mathscr{H}_{0}\left(\phi_{2}\right)$. It is clear that $\check{\phi}_{3}(w)=\check{\phi}_{1}(w)\left(\mathscr{H}_{0}\left(\phi_{2}\right)\right)(w)$. Let $a_{n}^{i}=\int_{0}^{\infty} \phi_{i}(t) L_{n}(t) e^{-t / 2} d t$. By Theorem 2.1 $\check{\phi}_{i}(w)=(1-w) \sum_{n} a_{n}^{i} w^{n}$ for $i=1,2,3$.

Since $\phi_{1} \in S^{+} \cap S$, by Theorem B $\lim _{w \rightarrow 1} \check{\phi}_{1}^{(k)}(w)=0$ for all $k \geq 0$ and since $\phi_{2} \in S^{+} \cap S$ by Theorem $4.1, \lim _{w \rightarrow-1}\left(\mathscr{H}_{0} \phi_{2}\right)^{-(k)}(w)=0$ for all $k \geq 0$. So $\lim _{w \rightarrow 1} \check{\phi}_{3}^{(k)}(w)=\lim _{w \rightarrow-1} \check{\phi}_{3}^{(k)}(w)=0$ for all $k \geq 0$, hence (see proof of Theorem 4.1) $\sum_{n} n^{k} a_{n}^{3}=0$ and $\sum_{n}(-1)^{n} n^{k} a_{n}^{3}=0$ for all $k \geq 0$. By the previous theorem, $\phi_{3} \in T$.

Finally, we propose the following problem (converse of the previous example).

Problem. Let $\phi \in T$, do there exist $\phi_{1}, \phi_{2} \in S^{+} \cap S$ such that $\phi=\phi_{1} * \mathscr{H}_{0}\left(\phi_{2}\right)$ ?

Notice that this problem is equivalent to the following one:

Let $f \in H(D)$ such that $f$ and all its derivatives are bounded in $D$ and $\lim _{w \rightarrow 1} f^{(k)}(w)=\lim _{w \rightarrow-1} f^{(k)}(w)=0$ for all $k \geq 0$. Do there exist two functionis $g, h \in H(D)$ bounded and with all its derivatives bounded in $D$ such that $\lim _{w \rightarrow 1} g^{(k)}(w)=0$ and $\lim _{w \rightarrow-1} h^{(k)}(w)=0$ for all $k \geq 0$ and $f=g \cdot h$ ? 


\section{ACKNOWLEDGMENT}

I wish to thank Professor J. Arias for his interest and advice.

\section{REFERENCES}

1. N. I. Akhiezer, The classical moment problem, Oliver and Boyd, Edinburgh, 1965.

2. R. P. Boas, The Stieltjes moment problem for functions of bounded variation, Bull. Amer. Math. Soc. 45 (1939), 399-404.

3. A. J. Duran, Laguerre expansions of tempered distributions and generalized functions, (to appear in J. Math. Anal. Appl).

4. N. F. Donoghue, Distribution and Fourier transform, Academic Press, New York 1969.

5. A. Erdelyi, ed., Tables of integral transforms, Volume 1, Mc-Graw Hill, New York 1954.

6. __ Higher transcendental functions, Volume 2, Mc-Graw Hill, New York 1953.

7. M. Guillemot-Teissier, Developpements des distributions en series de fonctions orthogonales. Series de Legendre et de Laguerre, Ann. Scuola Norm. Sup. Pisa (3) 25 (1971), 519-573.

8. G. H. Hardy, On Stieltjes "probleme des moments" (continued), in collected papers of G. M. Hardy Volume VII, Clarendon Press, Oxford 1979, 84-91.

9. G. Polya, Sur l'indetermination d'un theoreme voisin du probleme des moments, C. R. Acad. Sci. Paris 207 (1938), 708-711.

10. G. Sansone, Orthogonal functions, Interscience Publishers, New York 1959.

11. I. J. Schwatt, Operations with series, 2nd ed., Chelsea, New York.

12. J. A. Shoat and J. D. Tamarkin, The problem of moments, Amer. Math. Soc., Providence 1943.

13. T. J. Stieltjes, Recherches sur les fractions continues, Annales de la Faculte des Sciences de Toulousse (1) 8 (1894) T 1-122, (1) 9 A 5-47.

14. M. Valdivia, Interpolación en espacios de functiones holomorfas con desarrollos asintoticos, preprint.

Departamento de análisis Matemático, Facultad de Matemáticas, Universidad de Sevilla, Apart. 1160, 41080 Sevilla, Spain 